\title{
Application of three-dimensional printing technology and Plan-Do-Check-Act (PDCA) cycle in deviated nose correction
}

\author{
Tae-Hoon Lee, MD, PhD (i), Soonjoon Kim, MD (1) \\ Department of Otolaryngology-Head and Neck Surgery, Ulsan University Hospital, University of Ulsan College of Medicine, Ulsan, Rep. of Korea
}

Deviated nose correction, a challenging procedure, requires a strategic approach to achieve better results. The Plan-Do-Check-Act (PDCA) cycle can be adapted as a strategy. Three-dimensional (3D) scans and computer simulations enhance planning. Anterior septal reconstruction and support with titanium miniplates are helpful in correcting nasal and septal deviation correction. During surgery, checking with a customized 3D printing rhinoplasty guide is essential for realizing the simulated nose shape. A detailed assessment of nose shape and subjective evaluation of patient satisfaction are necessary during the follow-up period for continuous improvement.

Keywords: computer simulation; nasal septum; rhinoplasty; three-dimensional printing

\section{Introduction}

In rhinoplasty, deviated nose correction is one of the most challenging areas. Because a deviated nose is accompanied by severe and complex septal deviation, evaluation and correction play a vital role in corrective surgery [1]. The challenges in correcting nasal deviation are increased by asymmetric nasal bones and upper and lower lateral nasal cartilages. After surgery, the asymmetric forces of the soft tissue on each side cause the nose to bend again [2]. In addition, a variety of constraints worsen the outcome of the surgery for each patient, and so the learning curve to accurately identify them and acquire skills to cope with them appropriately is long.

The Plan-Do-Check-Act (PDCA) cycle is a method of managing production and quality in business administration [3]. It repeats the four steps of PDCA to improve the work continuously, and is also called the Plan-Do-Study-Act (PDSA) cycle, Deming cycle, and Shewhart cycle. It is most useful when starting a new project; developing a process, product, or service; or when continuous improvement is required. Although continuous improvement through execution and feedback on outcomes is a crucial principle, the success or failure of PDCA is often determined by planning capabilities.

Three-dimensional (3D) printing technology, a core technology in advanced industry, is expanding medical applications such as anatomical models for surgical preparation, customized surgical guides, implants and prostheses, and bioprinting of tissues and organs [4]. We introduce a strategic surgical method based on the PDCA cycle to continuously improve the results of deviated nose correction using 3D printing technology.

Received May 31, 2021; Revised June 9, 2021; Accepted June 10, 2021

Corresponding author: Tae-Hoon Lee

E-mail: thlee@uuh.ulsan.kr

This is an Open Access article distributed under the terms of the Creative Commons Attribution Non-Commercial License (http://creativecommons.org/licenses/by-nc/4.0), which permits unrestricted non-commercial use, distribution, and reproduction in any medium, provided the original work is properly cited.

Copyright @ 2021 Korean Society of Korean Cosmetic Surgery and Medicine (KSKCS \& KCCS). 


\section{Plan}

In planning rhinoplasty and patient communication, 3D simulation is beneficial. We perform a 3D scan using a Vectra ${ }^{\circledR} \mathrm{H} 2$ (Canfield Scientific, Parsippany, NJ, USA) scanner and virtual modeling using Sculptor ${ }^{\circledR}$ (Canfield Scientific) software (Fig. 1). At this time, we comprehensively reflect on the patient's needs and consider the feasibility of the plan. When a patient wants nasal augmentation, we also consider the available implant material and skin elasticity. We then adjust the simulated rhinoplasty images with the patient.

\section{Do}

Septal deviation correction is essential for correcting not only the deviated nose but also the septum. Because the deviation of the L-strut, which acts as a pillar of the nose, is open, we mainly use the anterior septal reconstruction method to correct it [5]. Leaving $1 \mathrm{~cm}$ to $1.5 \mathrm{~cm}$ of the dorsal cartilaginous septum, we separate the rest of the septal cartilages, and then use the straight part to create a new caudal septum and suture it with the dorsal septum to create a new L-strut. At this time, we adjust the size and position of the caudal septum in consideration of effects such as the septal extension graft, using a titanium

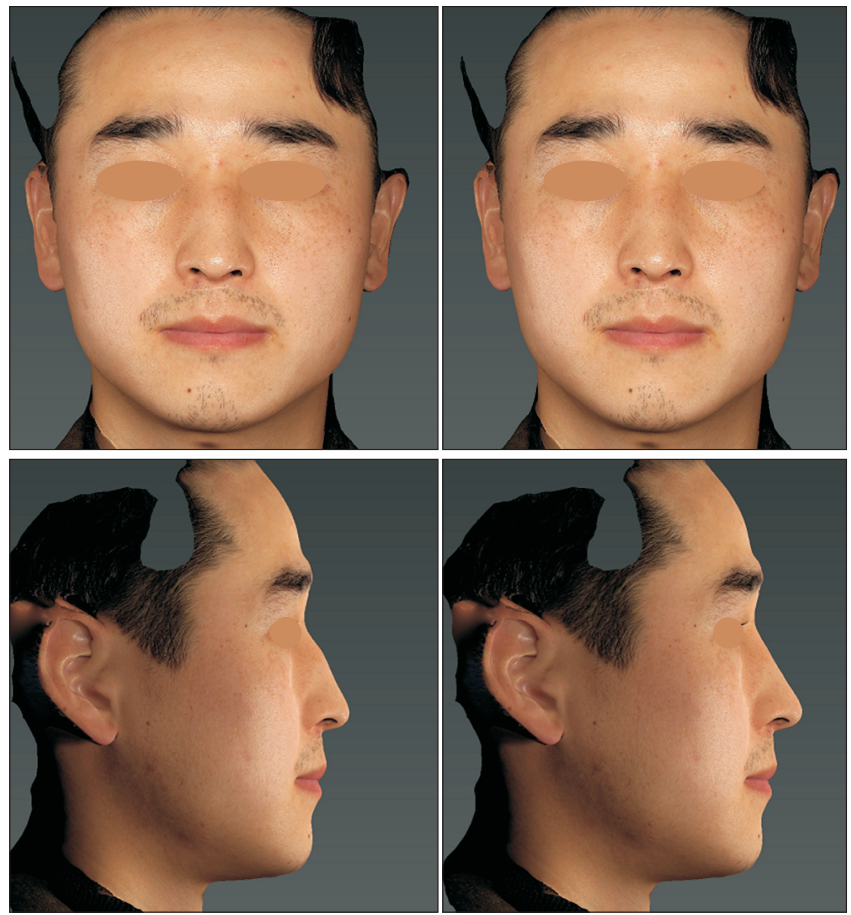

Fig. 1. Preoperative and simulated three-dimensional photographs: (left) preoperative, (right) simulation. miniplate to achieve stronger support (Fig. 2). Thereafter, we perform medial and lateral osteotomies to correct the nasal bony deviation and implement spreader grafts to correct the cartilaginous deviation. Alar cartilage is sutured to the L-strut to promote symmetry and centralization.

\section{Check}

Optical illusions may occur in the operating field due to the patient's posture, lighting, and drape, and confirmation bias increases with operation time. Therefore, we devised a rhinoplasty surgical guide to overcome these illusions [6]. We send simulation data to Nextcore Corp., Ltd. (Ulsan, Korea), a company specializing in 3D printing, where they produce customized rhinoplasty guides that match simulated nasal shapes. Design, manufacturing, and delivery usually takes two days. Aided by the medial canthus indicators and nasal alar holders in the rhinoplasty guide, we place it in the center of the face, marking the midline connecting the midpoint between both medial canthi and the middle of the Cupid's bow. We use this midline marking as a guide for osteotomies. We also found that the rhinoplasty guide is very useful for positioning a new L-strut acting as a nose pillar. In addition, we found it advantageous when checking the height and position of the nasal dorsum and tip (Fig. 3).

\section{Act}

Patient satisfaction with surgery and possible problems during follow-up are of the utmost importance in planning for improvement in subsequent surgery. Detailed operation records,

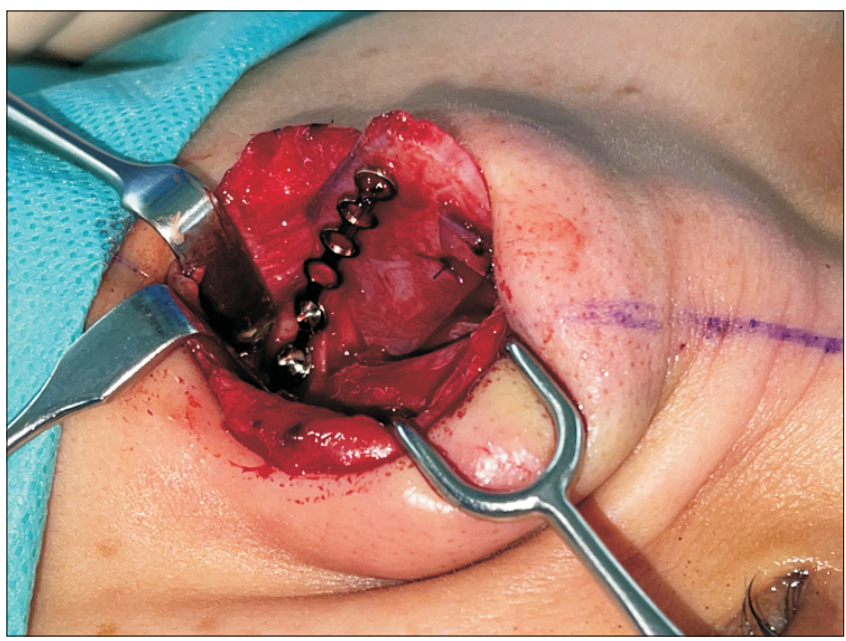

Fig. 2. A reconstructed L-strut supported by a titanium miniplate. 

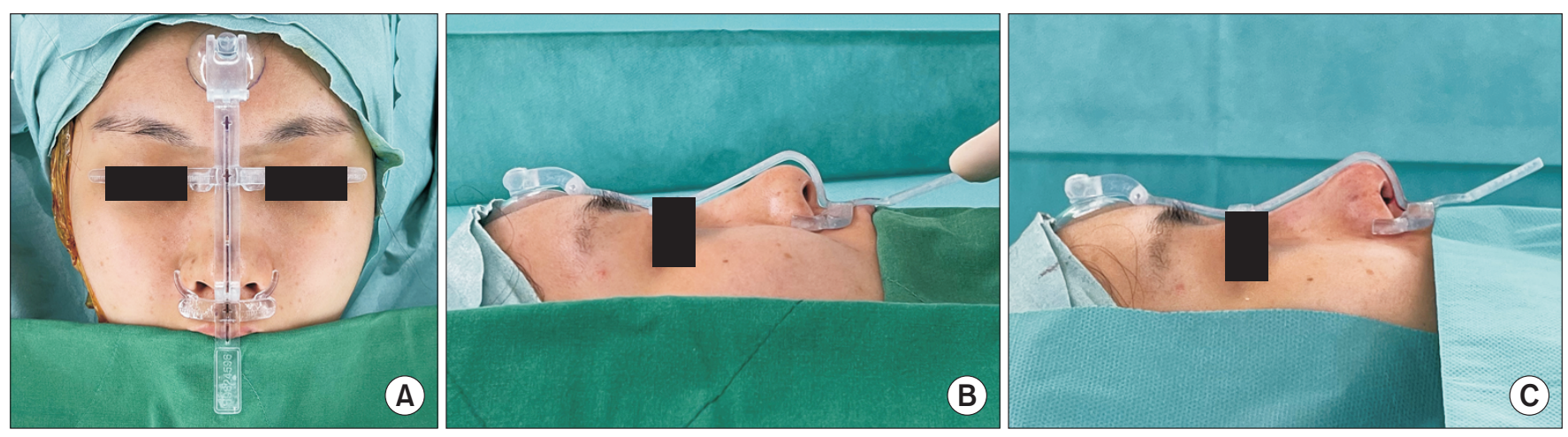

Fig. 3. Application of the customized rhinoplasty guide to check the straightening (A), height, and position of the nose during surgery (B: preoperative; C: postoperative).

detailed assessment of the shape of the nose, and subjective evaluation of patient satisfaction are beneficial for identifying problems. To this end, we take nasal photos before surgery and at 3, 6, and 12 months after surgery and conduct the Korean version of the Standardized Cosmesis and Health Nasal Outcomes Survey [7]. We also found it helpful to perform a postoperative 3D scan three months after surgery for comparison of preoperative rhinoplasty simulations and actual rhinoplasty results in 3D.

\section{Discussion}

Preoperative rhinoplastic simulation, by enhancing patient communication, is advantageous in improving surgical outcomes through unambiguous patient communication. However, it is seldom used in clinics, because in many cases the planned and actual surgical results do not exactly match, in which case simulations become the basis of complaints and may instead harm the surgeon. We recognized this problem and, to solve it, devised a customized rhinoplasty guide using $3 \mathrm{D}$ printing technology. The surgical guide has since improved the accuracy of surgery according to the simulated nose shape and further improved patient satisfaction through the simulation process and enhanced surgical results. The authors will conduct additional clinical trials on the effects of this customized rhinoplasty guide.

Titanium miniplates enhance septum stability, thereby improving long-term surgical outcomes [8]. In the deviated nose, asymmetry in the bone, cartilage, and soft tissue may cause redeformation after surgery. The strong septal support required to prevent redeformation is provided by the titanium miniplate. The accompanying disadvantage is that, in the case of bumping the nose, the miniplate and nose may bend, but this is easily corrected with closed reduction. The miniplate may be removed under local anesthesia around six months after rhinoplasty, when the nose shape is judged to be sufficiently stable. The use of titanium miniplates for nasal septal support is a new technique, and there is concern about its extrusion. Further clinical trials are necessary to analyze the usefulness and safety of titanium miniplates.

\section{Conclusion}

Deviated nose correction is a complicated operation that requires significant experience and a variety of skills. Therefore, a more strategic approach is necessary to improve the skills of rhinoplasty surgeons more efficiently. The PCDA process, which is currently overlooked in favor of the implementation of surgical techniques, deserves greater attention. Computer simulation and $3 \mathrm{D}$ printing technology hold great potential as invaluable aids for surgeons.

\section{Acknowledgments}

Each patient was given an opportunity to review the manuscript and consented to its publication

\section{Conflicts of interest}

The authors have nothing to disclose.

\section{References}

1. Rohrich RJ, Gunter JP, Deuber MA, Adams WP Jr. The deviated nose: optimizing results using a simplified classification and algorithmic approach. Plast Reconstr Surg 2002;110:1509- 
23.

2. Abu El-Wafa AM, Emara SS. Deviated nose: a systematic approach for correction. Plast Reconstr Surg Glob Open 2020;8:e3078.

3. Dewey J. The later works, 1925-1953. Carbondale, IL: Southern Illinois University Press; 1988.

4. Hsieh TY, Dedhia R, Cervenka B, Tollefson TT. 3D printing: current use in facial plastic and reconstructive surgery. Curr Opin Otolaryngol Head Neck Surg 2017;25:291-9.

5. Surowitz J, Lee MK, Most SP. Anterior septal reconstruction for treatment of severe caudal septal deviation: clinical severity and outcomes. Otolaryngol Head Neck Surg 2015;153:27-
33.

6. Choi JW, Kim MJ, Kang MK, Kim SC, Jeong WS, Kim DH, et al. Clinical application of a patient-specific, three-dimensional printing guide based on computer simulation for rhinoplasty. Plast Reconstr Surg 2020;145:365-74.

7. Moubayed SP, Ioannidis JPA, Saltychev M, Most SP. The 10item standardized cosmesis and health nasal outcomes survey (SCHNOS) for functional and cosmetic rhinoplasty. JAMA Facial Plast Surg 2018;20:37-42.

8. Mittermiller PA, Sheckter CC, Most SP. Efficacy and safety of titanium miniplates for patients undergoing septorhinoplasty. JAMA Facial Plast Surg 2018;20:82-4. 